\title{
Detection of low bone mineral density by dual energy $x$ ray absorptiometry in unsuspected suboptimally treated coeliac disease
}

\author{
J R F Walters, L M Banks, G P Butcher, C R Fowler
}

\begin{abstract}
Patients with coeliac disease may present with calcium malabsorption but it is unclear whether this results in longterm impairment of bone mineralisation. Dual energy $x$ ray absorptiometry (DXA) was used to study bone mineral density in 34 asymptomatic coeliac disease patients, treated with a gluten free diet for at least two years, and also in 10 newly diagnosed or untreated patients. As expected, untreated patients had low bone mineral density in all regions. In the 29 treated female coeliac disease patients, overall mean values for age adjusted bone mineral density expressed as $Z$ scores were normal although there were many patients with low values, particularly of the lumbar spine and total body. Scores in the postmenopausal patients were significantly worse than in the premenopausal patients and low mean $Z$ scores were found in the five treated male patients. The subjects who had reduced bone mineral density could not be predicted clinically but, despite being asymptomatic, were more likely to have subtotal or partial villous atrophy on small intestinal biopsy $(p<0.0275)$. In conclusion, although many treated coeliac disease patients have normal bone mineral density, suboptimally treated and newly diagnosed or untreated patients have osteopenia. To reduce the risk of osteoporotic fractures, it is recommended that bone mineral density be measured in all treated coeliac disease patients and those with osteopenia have a repeat intestinal biopsy to assess disease activity.
\end{abstract}

(Gut 1995; 37: 220-224)

Keywords: osteopenia, calcium malabsorption, villous atrophy, gluten free diet.

Departments of Medicine and Radiology, Hammersmith Hospital, Royal Postgraduate Medical School, London J R F Walters L M Banks G P Butcher C R Fowler

Correspondence to: Dr J R F Walters, Gastroenterology Unit, Royal Postgraduate Medical School, Du Cane Road London W12 0NN. Accepted for publication 7 December 1994 most actively absorbed, and also from the unabsorbed fatty acids, which bind calcium the intestinal lumen and may reduce dieta vitamin D absorption. ${ }^{2}$ Adherence to a strict gluten free diet will reverse the histological changes in the intestine and also the biochemical evidence of calcium malabsorption.
Evidence is now accumulating from preliminary studies, however, that suggests there may be longterm impairment of bone mineralisation in some otherwise healthy coeliac disease patients. ${ }^{3-9}$

Bone mineral density is now most easily assessed by duel energy $x$ ray absorptiometry (DXA), which is a rapid method to obtain precise and accurate values at selected sites such as the lumbar spine and femoral neck. Total body estimates of calcium, fat, and lean mass can also be obtained. ${ }^{10}$ Low bone mineral density (osteopenia) can now be detected cheaply with low radiation exposure, which enables screening of populations at risk, so that steps may be taken to prevent further loss of bone and subsequent osteoporotic fractures. ${ }^{11}$ In this paper, we report our studies using DXA to determine the prevalence of osteopenia in healthy, treated, adult coeliac disease patients under longterm follow up in our clinic and find that in many there is unpredicted osteopenia reflecting unsuspected suboptimal dietary treatment.

\section{Methods}

Selection of study groups

Fifty unselected outpatients under follow up for biopsy confirmed coeliac disease were invited to participate and were given a questionnaire asking about symptoms, diet, milk intake, exercise, smoking, and medication. Forty six agreed to have bone mineral density measurements performed. The results from one Asian patient, and one amputee were not analysed further. Eight patients diagnosed within two years of the study, a time chosen to allow for bone remineralisation on treatment, ${ }^{11}$ and two patients who were not receiving a gluten free diet formed a group of 'newly diagnosed/untreated' patients; the results from these 10 patients were analysed separately. The remaining 34 subjects $(29 \mathrm{~F}$ and $5 \mathrm{M}$ ) formed the main group of 'treated' patients.

The group of treated patients were all healthy white subjects in whom coeliac disease had been diagnosed more than two years previously by proximal small intestine biopsy and who had shown clinical and histological improvement on gluten withdrawal. They reported no current symptoms attributable to coeliac disease and all claimed to be strictly adherent to a gluten free diet. Patients' responses were confirmed regarding menopausal status, intake of milk, calcium 
TABLE I Details of the group of treated coeliac disease patients

\begin{tabular}{lll}
\hline & Female & Male \\
\hline Patients (n) & 29 & 5 \\
Age $(\mathrm{y})^{\star}$ & $52(14)$ & $56(15)$ \\
Weight $(\mathrm{kg})^{\star}$ & $63(14)$ & $58(14)$ \\
Height $(\mathrm{m})^{\star}$ & $1 \cdot 60(0 \cdot 08)$ & $1 \cdot 62(0 \cdot 10)$ \\
Body mass index $\left(\mathrm{kg} / \mathrm{m}^{2}\right)^{\star}$ & $24 \cdot 5(4 \cdot 3)$ & $21 \cdot 6(2 \cdot 8)$ \\
Time since diagnosis $(\mathrm{y}) \dagger$ & $16(2-47)$ & $17(3-30)$ \\
Calcium supplements & 9 & 1 \\
Vitamin D supplements & 2 & 1 \\
Other significant diseases $\ddagger$ & 8 & 1 \\
Premenopausal & 10 & 0 \\
Perimenopausal with HRT & 3 & 0 \\
Postmenopausal: & 14 & \\
$\quad$ Never received HRT & 2 & 0 \\
Delayed HRT & & 0 \\
\hline
\end{tabular}

${ }^{\star}$ Mean (SD); †median (range); łdiabetes (3), alcohol abuse (2), thyroxine therapy (3), epilepsy (1), previous prednisolone therapy for asthma (2) or menigioma (1). Two diseases occurred in three patients.

HRT = hormone replacement therapy.

supplements, vitamin $\mathrm{D}$, hormone replacement therapy with oestrogen, other treatments, and smoking history. Table I shows these patients' details. Normal values of serum calcium, alkaline phosphatase, and albumin were present. Small bowel biopsy specimens had been obtained within two years from five of these patients; all other patients who had bone mineral density measurements were invited by letter to have a repeat biopsy. Biopsy specimens were obtained from another 12 patients.

\section{Bone mineral density measurements}

Measurements were made by DXA on the Lunar DPX-L machine using the manufac-
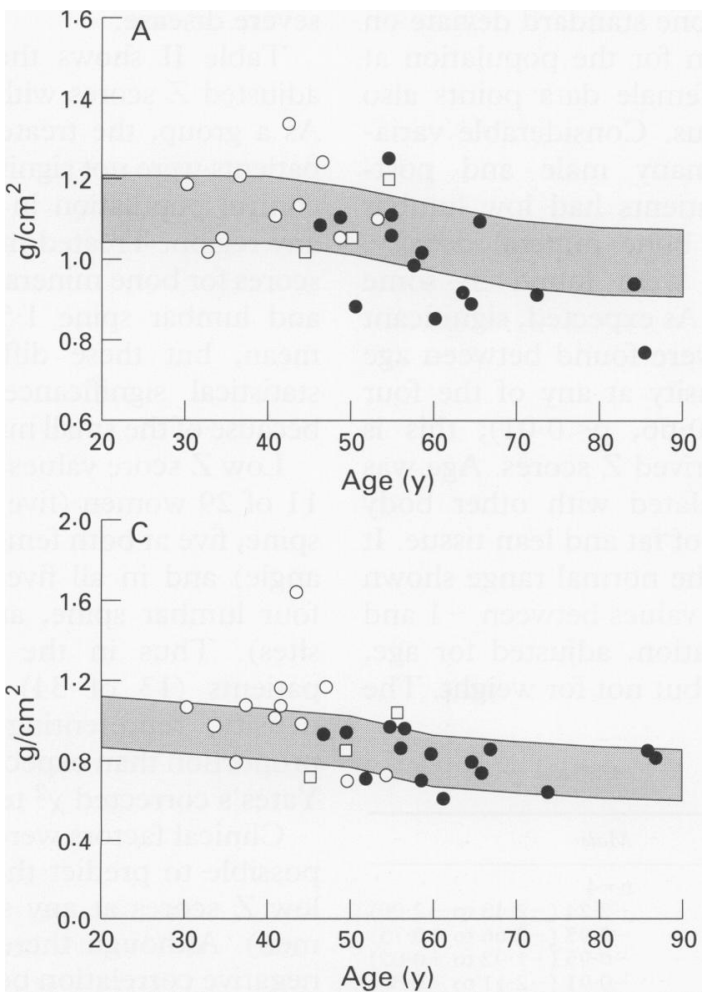

Figure 1: Bone mineral density values obtained by DXA in 29 treated female patients. (A) Total body, (B) lumbar spine (L2-L4), (C) femoral neck, and (D) Ward's triangle region of the femoral neck. Premenopausal patients are shown as $\bigcirc$, postmenopausal patients not receiving hormone replacement as $\mathbf{O}$, and the perimenopausal patients receiving hormone replacement as $\square$. The shaded area shows one standard deviation on either side of the age adjusted mean bone mineral density. This represents $Z$ score values between -1 and +1 for a subject of average weight but does not show the weight adjusted $Z$ score for heavier or lighter subjects.

turer's protocols. Results of bone mineral density in the total body, lumbar spine (L2-L4), femoral neck, and the Ward's triangle region of the femur were analysed with the current analysis software (version 1.3z). These were expressed both in terms of absolute areal bone mineral density values $\left(\mathrm{g} / \mathrm{cm}^{2}\right)$ and as $\mathrm{Z}$ scores (normalised standard deviate) corrected for age, sex, ethnic origin, and weight using normal British standard values. Values were also obtained by DXA for total body fat and lean tissue and the subject's height and weight recorded.

\section{Results}

\section{Newly diagnosed/untreated patients}

As expected, in our group of 10 patients who had been recently diagnosed, or who were not receiving a gluten free diet, reduced values for bone mineral density were common. Mean age adjusted $\mathrm{Z}$ scores were low in both men and women at all sites with the reduction in total body mineral density being most noticeable (see Table II). All these reductions were highly significant $(p<0.001$ for total body, lumbar spine, and femoral neck; $\mathrm{p}<0.01$ for Ward's triangle; $\mathrm{n}=10$ men and women). Some of these patients had hypocalcaemia, reduced serum 25-hydroxyvitamin $\mathrm{D}$, and increased parathyroid hormone concentrations suggesting biochemical osteomalacia; bone biopsy was not performed to establish the histological extent that defective bone mineralisation contributed to the osteopenia.
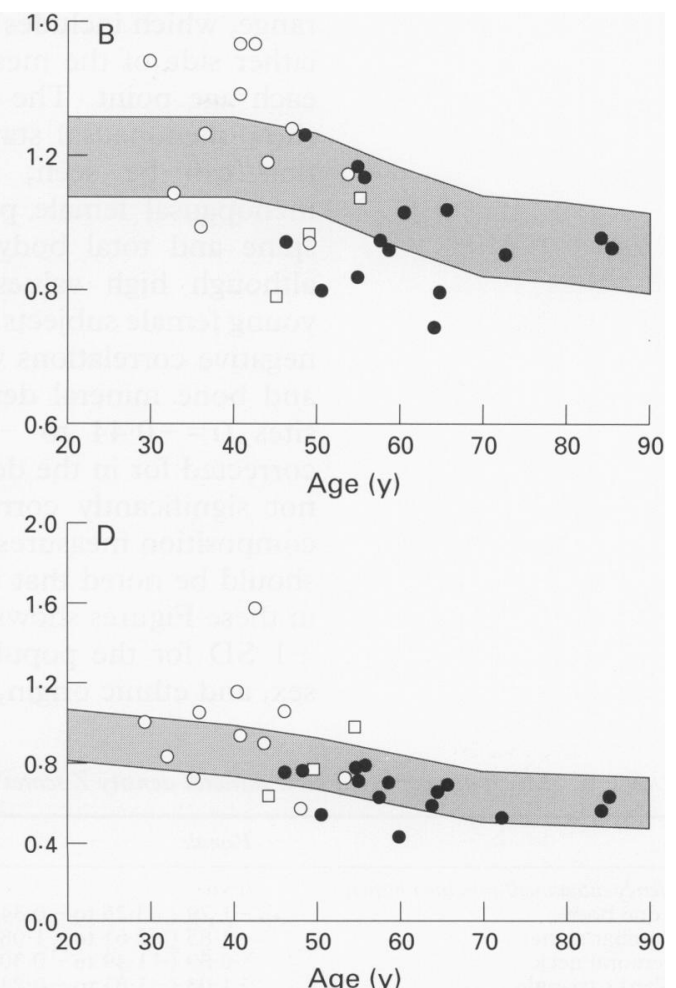

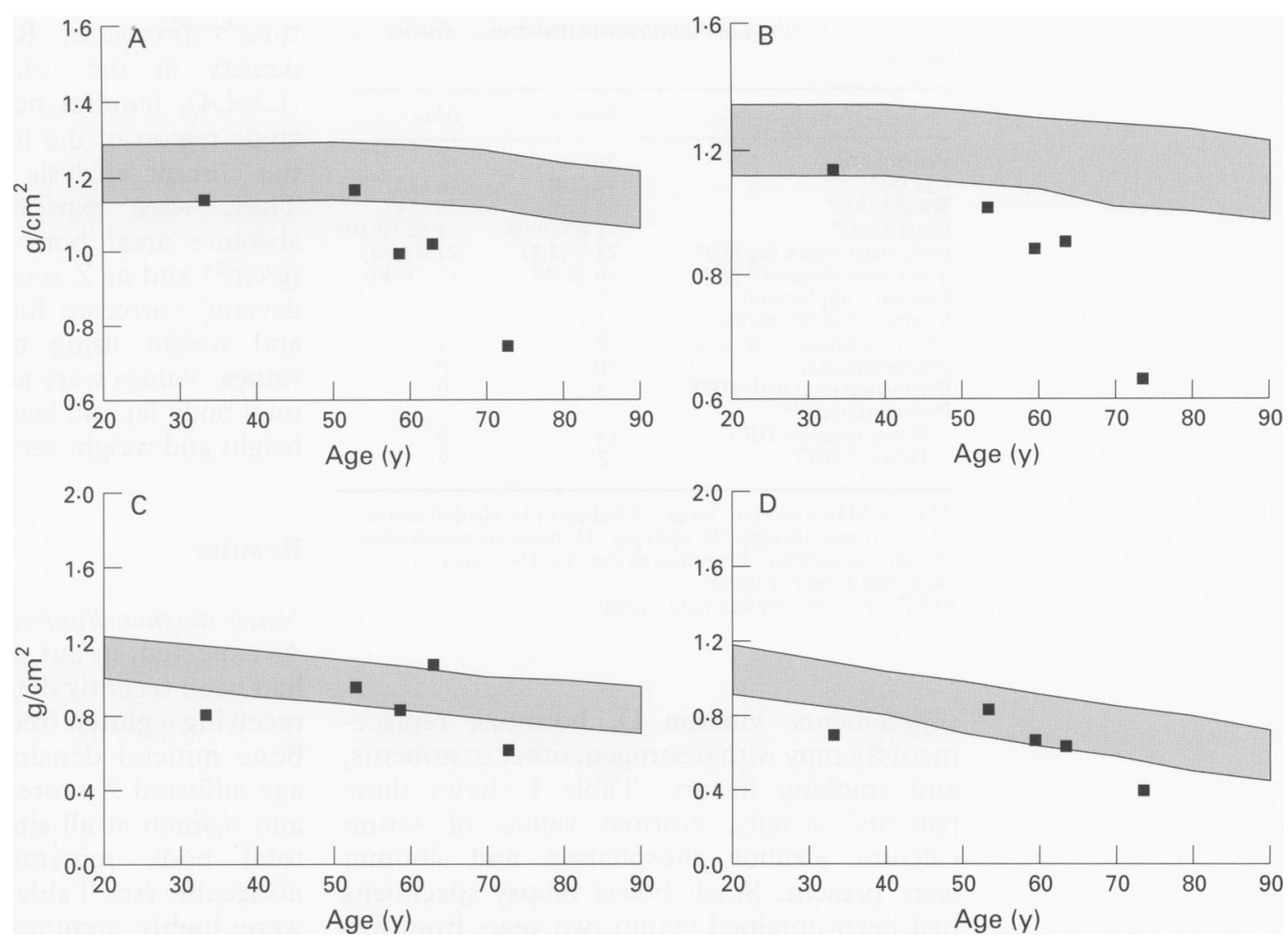

Figure 2: Bone mineral density in five treated male patients. (A) Total body, (B) lumbar spine (L2-L4), (C) femoral neck, and $(D)$ Ward's triangle. One standard deviation on either side of the age adjusted mean value is shown by the shaded area.

\section{Treated patients}

The individual bone mineral density values obtained by DXA in the 34 treated patients for total body, lumbar spine, femoral neck, and Ward's triangle are shown in Figs 1 and 2 for female and male subjects respectively. These values are plotted against age and show the range, which includes one standard deviate on either side of the mean for the population at each age point. The female data points also show menopausal status. Considerable variation can be seen; many male and postmenopausal female patients had low lumbar spine and total body bone mineral density, although high values were found in some young female subjects. As expected, significant negative correlations were found between age and bone mineral density at any of the four sites $(r=-0.44$ to $-0.66, \mathrm{p}<0.01)$; this is corrected for in the derived $\mathrm{Z}$ scores. Age was not significantly correlated with other body composition measures of fat and lean tissue. It should be noted that the normal range shown in these Figures shows values between -1 and +1 SD for the population, adjusted for age, sex, and ethnic origin, but not for weight. The

TABLE II Mean age corrected bone mineral density $Z$ scores

\begin{tabular}{lll}
\hline & Female & Male \\
\hline Newly diagnosed/untreated patients & $\mathrm{n}=6$ & $\mathrm{n}=4$ \\
Total body & $-1.79(-3.25$ to -0.34$)$ & $-2.24(-2.48$ to $-2 \cdot 00)$ \\
Lumbar spine & $-1.85(-2.61$ to -1.08$)$ & $-0.95(-2.66$ to +0.75$)$ \\
Femoral neck & $-0.89(-1.49$ to -0.30$)$ & $-0.95(-1.92$ to +0.02$)$ \\
Ward's triangle & $-1.03(-1.81$ to -0.24$)$ & $-0.91(-2.11$ to +0.30$)$ \\
Treated patients & $\mathrm{n}=29$ & $\mathrm{n}=5$ \\
Total body & $-0.18(-0.59$ to +0.23$)$ & $-1.50(-3.1 .9$ to +0.19$)$ \\
Lumbar spine & $-0.05(-0.64$ to +0.54$)$ & $-1.83(-3.84$ to +0.18$)$ \\
Femoral neck & $+0.27(-0.25$ to +0.79$)$ & $-0.42(-2.20$ to +1.35$)$ \\
Ward's triangle & $+0.14(-0.38$ to +0.67$)$ & $-0.71(-1.58$ to +0.17$)$
\end{tabular}

${ }^{\star}$ Figures in parentheses show the $95 \%$ confidence limits for the mean $\mathrm{Z}$ scores. individual $\mathrm{Z}$ scores are also adjusted for weight, which resulted in an upward correction of the $Z$ score to above -1 in several of the small female patients. As a group, the male patients were particularly small in size and also in number, it may be that men who undergo follow up are self selected for particularly severe disease.

Table II shows the mean age and weight adjusted $\mathrm{Z}$ scores with $95 \%$ confidence limits. As a group, the treated female coeliac disease patients were not significantly different from the control population in bone mineral density at any region. Treated male patients had mean $\mathrm{Z}$ scores for bone mineral density in the total body and lumbar spine 1.5 SD below the normal mean, but these differences failed to reach statistical significance at the $p=0.05$ value because of the small number of subjects studied.

Low $\mathrm{Z}$ score values below -1 were found in 11 of 29 women (five total body, nine lumbar spine, five at both femoral neck and Ward's triangle) and in all five men (three total body, four lumbar spine, and two at both femoral sites). Thus in the lumbar spine, $38 \%$ of patients (13 of 34 ) had low bone mineral density, representing a significantly greater proportion than expected by chance $(p=0.011$, Yates's corrected $\chi^{2}$ test).

Clinical factors were analysed to see if it was possible to predict the group of patients with low $\mathrm{Z}$ scores at any site (11 women and five men). Although there was a significant weak negative correlation between the length of time with coeliac disease and absolute total body bone mineral density $(r=-0.42, \mathrm{p}<0.02)$, this reflected the bone loss with age in those with the longest duration of disease and no correlation was found when values were expressed 
TABLE III Small intestinal histological assessment in treated coeliac disease patients with reduced or normal bone mineral density

\begin{tabular}{lll}
\hline & $\begin{array}{l}\text { Osteopenic } \\
\text { group }\end{array}$ & $\begin{array}{l}\text { Normal } \\
\text { group }\end{array}$ \\
\hline Patients (n) & 16 & 18 \\
Number with biopsies within 2 years & 8 & 9 \\
Small intestinal histology & 7 & 2 \\
$\quad$ Subtotal or partial villous atrophy & 7 & 7 \\
$\quad$ Normal & 1 &
\end{tabular}

The osteopenic group included all the patients in whom any bone mineral density $\mathrm{Z}$ score was below -1 . The normal group had no value more than 1 standard deviation below the age adjusted mean. The difference between the groups in the proportion with abnormal histological assessment was significant $\left(p<0 \cdot 0275\right.$, Yates's corrected $\chi^{2}$ test $)$.

as age corrected $\mathrm{Z}$ scores. Significantly lower $\mathrm{Z}$ scores for total body and lumbar spine bone mineral density were found in postmenopausal women compared with premenopausal women $(p=0.02$ and 0.03 respectively). No correlations were apparent with reported calcium intake, smoking or exercise. The numbers of patients receiving hormone replacement therapy were too small and varied for meaningful analysis.

Recent small intestinal histological examination, performed while receiving a gluten free diet and within two years of the bone mineral density measurements, was obtained in eight of 16 patients with low $\mathrm{Z}$ scores and in nine of 18 patients with entirely normal $Z$ scores. Table III shows that the patients with low bone mineral density were significantly more likely to have abnormal histological tests $(p<0.0275$, Yates's corrected $\chi^{2}$ test). This represents an odds ratio of 24.5 for the association of osteopenia and villous atrophy.

\section{Discussion}

In this study of bone mineral density in coeliac disease using DXA, osteopenia has been shown in many asymptomatic coeliac patients receiving treatment with a gluten free diet, although overall, the mean values did not differ from age and sex matched controls. Low bone mineral density was confirmed in a group of newly diagnosed or non-compliant adult coeliac disease patients but, most importantly, we showed that the treated patients with low bone mineral density had persistently abnormal small intestinal biopsies presumably reflecting suboptimal treatment.

Our results extend some earlier studies, which used other, less precise methods to detect osteopenia. Bone mineral content, measured in the spine and forearm by dual and single photon absorptiometry respectively, was found to be significantly decreased in treated adult coeliac disease patients with mean values 7 to $13 \%$ lower than normal. 5 In another study of teenagers who had been treated for over 10 years, normal forearm mineral density was found by single photon absorptiometry. ${ }^{4}$ In newly diagnosed patients, reduced bone mineralisation has been clearly shown, but was reported not to have corrected after treatment with a gluten free diet for 12 months. ${ }^{3}$ Significant improvement, however, of radial bone mineral content in children after treatment with gluten free diets for over a year has recently been shown. ${ }^{9}$

Data using DXA in treated adult coeliac disease patients have been reported in preliminary abstracts by other groups and are in line with the present findings. Using the Hologic DXA machine and normal values in a similar group of coeliac disease patients, McFarlane et al showed that $42 \%$ had bone mineral density $\mathrm{Z}$ score values below one and $20 \%$ had values under two. ${ }^{6}$ In a study from Finland, Janatuinen et al found a larger proportion of patients with low lumbar bone mineral density (32 of 56 with $\mathrm{Z}$ scores below -1 ). ${ }^{7}$ In an Argentinian study using Lunar DXA equipment, low mean values for spinal and total body bone mineral density were shown in untreated coeliac disease patients, including those who were asymptomatic; again the mean values for treated patients were not different from controls, but $30 \%$ had low values. ${ }^{8}$

Data from all these studies suggest that the proportion of coeliac disease patients with low bone mineral density is about twice that expected, so giving a greater yield of osteopenic subjects than in screening studies of most other 'high risk' groups. The importance of these findings is that the risk of subsequent osteoporotic fracture has been shown to double for each decrease in bone mineral density of one standard deviation. ${ }^{12}$ Young subjects with low $\mathrm{Z}$ scores are not at risk of immediate fracture, but as they will continue to lose bone with aging, they will reach the values of bone mineral density associated with increased fractures at an earlier time than someone with average bone mineral density. The rationale for screening for osteopenia is to be able to target therapeutic interventions to prevent further bone loss, or reverse some of the existing changes, before the risk of fracture becomes significant. ${ }^{11}$ Direct measurement of bone mineral density by DXA is necessary to detect those patients requiring further investigation and treatment as repeat small bowel biopsy was the only clinical factor we found that was predictive of osteopenia. The association of abnormal histology on repeat biopsy with osteopenia was statistically significant in our treated coeliac disease patients despite the comparatively small number of patients (17) that could be studied. Only $50 \%$ of the patients overall were prepared to have a repeat biopsy, which reflects that they were asymptomatic and considered their adherence to diet to be excellent; we did not think that it would be possible to achieve repeat histological examination in all the patients. We recognise that this could have introduced some bias into the study and await with interest reports of similar cohorts of treated coeliac disease patients to confirm these findings.

Unrecognised lapses from a gluten free diet seem to be the most probable cause of the low bone mineral density in coeliac disease patients. In untreated or non-compliant patients, very low values for bone mineralisation are usual; reduction in the total intestinal mucosa surface area for absorption is obvious in this group. In patients with partially treated, 
subclinical disease, less pronounced changes occur, but these will affect predominantly the mature duodenal villous cells, which are those where the proteins responsible for calcium absorption are located. ${ }^{213}$ Loss of these cells will impair the absorption of calcium, increase faecal losses, and produce secondary hyperparathyroidism, which leads to bone demineralisation.

As most ambulatory subjects such as our patients synthesise sufficient vitamin $D$ in the skin with only moderate sun exposure, malabsorption of vitamin $D$ is less important, but stores may be used up faster as a result of the hyperparathyroidism. ${ }^{14}$ The vitamin $\mathrm{D}$ receptor is demonstrable in biopsy specimens from patients with active coeliac disease ${ }^{15}$ but resistance to the actions of vitamin $\mathrm{D}$ has been suggested. The cytosolic vitamin $\mathrm{D}$ dependent calcium binding protein, now known as calbindin-D9k, was almost undetectable in biopsy specimens from active coeliac disease, and in treated coeliac disease patients with no other evidence of villous atrophy, median values were only $26 \%$ of the control group. ${ }^{16} \mathrm{We}$ have recently cloned human calbindin-D $9 \mathrm{k},{ }^{17}$ and are now studying the transcriptional control of this key protein in humans.

When low bone mineral density is discovered in a coeliac patient, our data indicate that the adherence to the gluten free diet needs reviewing, even though the patient may be asymptomatic, and that a repeat small intestinal biopsy is likely to show persistent changes. Whether endomysial, reticulin or gliadin antibodies may be better to assess compliance is still uncertain. Osteopenia is another reason to insist on strict compliance with a gluten free diet, even in patients with few symptoms. Indeed, osteoporotic fractures are more likely to be a problem in coeliac patients than the other longterm complications such as intestinal lymphoma, which hitherto has been the main reason for insistence on more than symptomatic treatment. ${ }^{1}$ It is also of interest that a study that looked at patients with clinical osteoporosis found the incidence of unsuspected, asymptomatic coeliac disease to be roughly 10 -fold higher than expected. ${ }^{18}$

Although we recommend calcium supplementation in these coeliac patients, we do not know whether this alone may be effective in reducing bone mineral loss, as it seems to be in general in postmenopausal women with osteopenia. ${ }^{19}$ Calcium carbonate supplements are safe and effective, increasing net calcium absorption through passive absorption ${ }^{20}$ and with adequate physiological amounts of vitamin $\mathrm{D}$, as found in multivitamin preparations, will prevent hip fractures in elderly women. ${ }^{21}$ Hormone replacement therapy, however, is the most effective means to delay the considerable loss of bone mineral in women, 22 is known to be effective at preventing bone loss in patients with inflammatory bowel disease, ${ }^{23}$ and should be advocated in coeliac patients. Most importantly, we recommend screening bone mineral density in all coeliac patients to detect those at most risk so that these preventative measures may be directed to those patients with osteopenia who will then probably be found to have unsuspected active disease.

We thank Professor $\mathrm{H}$ Hodgson and Dr J Calam for including patients under their follow up in this study. An abstract of the preliminary findings was presented at the British Society for Gastroenterology (Gut 1992; 33 (suppl 2): S48). The study was supported by a grant from the Special Trustees of the Hammersmith and Queen Charlotte's Special Health Authority.

1 Trier JS. Celiac sprue. $N$ Engl f Med 1991; 325: 1709-19.

2 Walters JRF. Absorption and malabsorption of calcium and vitamin D. In: Bouchier IAD, Hodgson HJF, Allan RN, Keighley MRB, eds. Gastroenterology: clinical science and practice. 2nd ed. London: Baillière Tindall, 1993: 433-47.

3 Caraceni MP, Molteni N, Bardella MT, Ortolani S, Nogara A, Bianchi PA. Bone and mineral metabolism in adult celiac disease. Am $\mathcal{F}$ Gastroenterol 1988; 83: 274-7.

4 Molteni N, Caraceni MP, Bardella MT, Ortolani S, Gandolini GG, Bianchi P. Bone mineral density in adult celiac patients and the effect of gluten-free diet from childhood. Am 7 Gastroenterol 1990; 85: 51-3.

5 Bodé S, Hassager C, Gudmand-Hoyer E, Christiansen C. Body composition and calcium metabolism in adult treated coeliac disease. Gut 1991; 32: 1342-5.

6 McFarlane X, Bhalla A, Morgan L, Reeves D, Robertson DAF. Osteoporosis: a frequent finding in treated adult coeliac disease. Gut 1992; 33 (suppl 2): S48.

7 Janatuinen E, Alhava E, Arnarainen I, Julkunen R, Kosma VM, Kröger M, et al. Bone density and histomorphometry in adult coeliac disease. Gastroenterology 1992; 102: A216.

8 Mazure R, Vazquez H, Gonzalez D, Mautalen C, Boerr L, Bai JC. Low bone mineral density (BMD) in asymptomatic adult patients with untreated celiac disease. Gastroenterology 1993; 104: A264.

9 Mora S, Weber G, Barera G, Bellini A, Pasolini D, Prinster C, et al. Effect of gluten-free diet on bone mineral content in growing patients with celiac disease. Am $\mathcal{f}$ Clin Nutr 1993; 57: 224-8.

10 Johnston CC, Slemenda CW, Melton LJ. Clinical use of bone densitometry. $N$ Engl f Med 1991; 324: 1105-9.

11 Riggs BL, Melton LJ III. The prevention and treatment of osteoporosis. N Engl f Med 1992; 327: 620-7.

12 Melton LJ, Eddy DM, Johnston CC. Screening for osteoporosis. Ann Intern Med 1990; 112: 516-28.

13 Howard A, Legon S, Walters JRF. Human and rat intestinal plasma membrane calcium pump isoforms. Am $\mathcal{F}$ Physiol 1993; 265: G917-25.

14 Clements MR, Davies M, Hayes ME, Hickey CD, Lumb GA, Mawer EB, et al. The role of 1,25-dihydroxyvitamin $\mathrm{GA}$, Mawer EB, et al. The role of 1,25-dihydroxyvitamin $\mathrm{D}$ in the mechanism of acquired

15 Colston KW, Mackay AG, Finlayson C, Wu JCY, Maxwell JD. Localisation of vitamin $D$ receptor in normal human duodenum and in patients with coeliac disease. Gut 1994; 35: $1219-25$.

16 Staun M, Jarnum S. Measurement of the 10000 -molecular weight calcium-binding protein in small-intestinal biopsy specimens from patients with malabsorption syndromes. Scand $\mathcal{F}$ Gastroenterol 1988; 23: 827-32.

17 Howard A, Legon S, Spurr NK, Walters JRF. Molecular cloning and chromosomal assignment of human calbindin-D9k. Biochem Biophys Res Commun 1992; 185: $663-9$.

18 Lindh E, Ljunghall S, Larsson K, Lavö B. Screening for antibodies against gliadin in patients with osteoporosis. $\mathcal{J}$ Intern Med 1992; 231: 403-6.

19 Dawson-Hughes B, Dallal GE, Krall EA, Sadowski L, Sahyoun N, Tannenbaum S. A controlled trial of the effect of calcium supplementation on bone density in postmenopausal women. $N$ Engl fु Med 1990; 323: 878-83.

20 Sheikh MS, SantaAna CA, Nicar MJ, Schiller LR, Fordtran JS. Gastrointestinal absorption of calcium from milk and calcium salts. N Engl F Med 1987; 317: 532-6.

21 Chapuy MC, Arlot ME, Duboeuf F, Brun J, Crouzet B, Arnaud S, et al. Vitamin D3 and calcium to prevent hip Arnaud S, et al. Vitamin D3 and calcium to prevent hip fractures

22 Prince RL, Smith M, Dick IM, Price RI, Garcla-Webb P, Henderson NK, et al. Prevention of postmenopausal osteoporosis. A comparative study of exercise, calcium supplementation, and hormone-replacement therapy. $N$ Engl f Med 1991; 325: 1189-95.

23 Clements D, Compston JE, Evans WD, Rhodes J. Hormone replacement therapy prevents bone loss in patients with inflammatory bowel disease. Gut 1993; 34: 1543-6. 